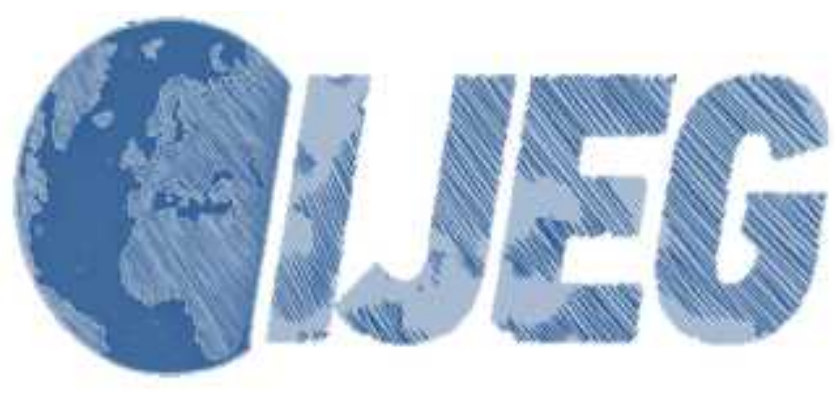

International Journal of Engineering and Geosciences (IJEG),

Vol;2, Issue;01, pp. 9-16, February, 2017, ISSN 2548-0960, Turkey,

DOI: 10.26833 /ijeg. 286003

\title{
COMPARING UNIFORM AND RANDOM DATA REDUCTION METHODS FOR DTM ACCURACY
}

\author{
Yilmaz, M., ${ }^{1 *}$ Uysal, M., ${ }^{1}$ \\ ${ }^{1}$ Afyon Kocatepe University, Engineering Faculty, Department of Geomatic Engineering, TR-03200 Afyonkarahisar, \\ Turkey (mustafayilmaz, muysal@aku.edu.tr)
}

*Corresponding Author, Received: 16/01/2017, $\quad$ Accepted: 28/01/2017

\begin{abstract}
The digital cartographic representation of the elevation of the earth's surface created from discrete elevation points is defined as a digital terrain model (DTM). DTMs have been used in a wide range of applications, such as civil planning, flood control, transportation design, navigation, natural hazard risk assessment, hydraulic simulation, visibility analysis of the terrain, topographic change quantification, and forest characterization. Remote sensing, laser scanning, and radar interferometry become efficient sources for constructing high-accuracy DTMs by the developments in data processing technologies. The accuracy, the density, and the spatial distribution of elevation points, the terrain surface characteristics, and the interpolation methods have an influence on the accuracy of DTMs. In this study, uniform and random data reduction methods are compared for DTMs generated from airborne Light Detection and Ranging (LiDAR) data. The airborne LiDAR data set is reduced to subsets by using uniform and random methods, representing the $75 \%, 50 \%$, and $25 \%$ of the original data set. Over the Mount St. Helens in southwest Washington State as the test area, DTM constructed from the original airborne LiDAR data set is compared with DTMs interpolated from reduced data sets by Kriging interpolation method. The results show that uniform data reduction method can be used to reduce the LiDAR datasets to $50 \%$ density level while still maintaining the quality of DTM.
\end{abstract}

Keywords: DTM, LiDAR, Uniform, Random, Kriging. 


\section{INTRODUCTION}

A digital elevation model (DEM) is defined as the digital cartographic representation of the elevation of the earth's surface in all its forms. The generic DEM normally implies elevations of the terrain void of vegetation and man-made features. This bare-earth DEM is is generally synonymous with a digital terrain model (DTM). DTMs frequently incorporate the elevation of the significant topographic features of the land, plus mass points and breaklines that are irregularly spaced so as to better characterize the true shape of the bare earth terrain (Maune et al., 2007). DTMs provide a threedimensional (3D) representation of the bare earth/underlying terrain of the earth's surface that contains elevations of topography (ridgelines, stream courses, breaklines, etc.) where vegetation, buildings, and other non-ground objects have been removed. DTMs have found wide application in all geosciences and engineering tasks such as: civil planning, mine engineering, military purposes, landscape design, urban planning, environmental protection, forest characterization, hydraulic simulation, visibility analysis of the terrain, surface modelling, topographic change quantification, volume computation, geomorphological extraction, satellite imagery interpretation, cartographic presentation, and geographical analysis ( $\mathrm{Li}$ et al., 2005; Tarolli et al., 2009; Cavalli and Tarolli, 2011). DTMs can be derived by field surveying, photogrammetry or cartographic digitization of existing topographic maps. Besides the conventional methods for creating DTMs, new technologies such as satellite remote sensing, radar interferometry and airborne laser scanning revolutionize the construction of high quality DTMs in a cost-effective manner. Due to advancements in reliability and spatial resolution over the past decades, airborne Light Detection and Ranging (LiDAR) is becoming the privileged data acquisition technique for high-resolution and high-accuracy DTMs over large areas owing to providing 3D non-uniformly spaced dense point information very effectively (Ma and Meyer, 2005; Liu, 2008; Vianello et al., 2009; Razak et al., 2011; ArabSedze et al., 2014, Polat and Uysal, 2015; Yan et al., 2015). LiDAR has become a well-established resource used to enhance spatial knowledge of the topography in order to construct DTMs while preserving high frequencies of the relief. The spatial distribution of usable data points is expected to be uniform for DTM construction in a broad application spectrum. Although, LiDAR do not produce regularly gridded points. The output of a LiDAR survey is a point cloud of hundreds of millions or billions of sample points representing the feature height. Each laser point is randomly located. In many cases, not all points may be required for defining the terrain surface. Therefore, the raw point clouds need to be processed (filtering and interpolation) in order to provide an approximation to a real-world continuous surface (Garnero and Godone, 2013).

The accuracy of the features derived from DTMs depends on several factors originating from: (i) the accuracy, the density, and the spatial distribution of elevation points, (ii) the interpolation methods, (iii) the terrain surface characteristics (Gong et al., 2000; Chen and Yue, 2010; Liu et al., 2011; Sailer et al., 2014). The first two factors are clearly errors (the objective problems with measurement/estimation), whereas the third should be considered a matter of uncertainty (less tangible issues). Alternatively, the first can be regarded as data-based, being strictly concerned with the source data, while the second and the third are model-based, being concerned with how well the resulting DTM approximates the real physiography (Fisher and Tate, 2006). There has been extensive literature about these factors: the accuracy of data acquisition (Hodgson and Bresnahan, 2004; Rayburg et al., 2009; Mukherjee at al., 2013; Dorn et al., 2014); the data density (Aguilar et al., 2005; Chaplot et al., 2006; Liu et al., 2007); the spatial distribution of source data (Erdogan, 2010; Gumus and Sen, 2013; Fassnacht et al., 2014); the interpolation process (Yilmaz, 2007; Chen and Li, 2012; Arun, 2013; Tan and $\mathrm{Xu}, 2014$ ); the terrain features (Aguilar et al., 2007; Aguilar and Mills, 2008; Chu et al., 2014).

The contemporary airborne LiDAR systems can operate between 150.000 to 400.000 laser pulses per second, where achieved density (measurement resolution) exceeds 10 points per square meter (Renslow, 2012). Nowadays, there are even $1 \mathrm{GHz}(1.000 .000$ laser pulses) airborne LiDAR sensors available. The use of LiDAR has rapidly become a standard source of elevation data for building high quality DTMs. The DTM resolution has increased dramatically in the recent years as a consequence of higher LiDAR point densities. Modern LiDAR sensors allow simultaneously capturing topographic and bathymetric details from large geographical areas at the price of a highly increased data volume. When DTM of different resolution is required the common technique is removing data to produce a coarser resolution data set. Besides, the production of different horizontal resolution DTM from the same data source is important for predicting scale dependent environmental variables. The use of LiDAR offers the flexibility needed to produce multiple horizontal resolutions of DTM from the same data source. However, the high-density LiDAR data lead to a significant increase in the data volume, imposing challenges with respect to data storage, processing, and manipulation for producing DTM. Because of the copious number of LiDAR spot elevations returned on an areal basis, the effects of data density reduction on DTM of various horizontal resolutions is worthy of study, particularly for landscape scale studies. With a data reduction, a more manageable and operationally sized elevation data set is possible (Anderson et al., 2006). Therefore, terrain data reduction (achieving an adjustment between density of data and volume of data) without losing relevant geometric details has become a research topic while constructing DTMs. However, there are particularly limited studies about data reduction for DTMs (e.g., Anderson et al., 2005; Anderson et al., 2006; Liu et al., 2007; Liu and Zhang, 2008; Immelman and Scheepers, 2011). The main objectives of this study are to:

- Evaluate the effect of the data reduction algorithms on the accuracy of LiDAR-derived DTM construction.

- Examine to what extent a set of LiDAR data can be reduced while maintaining effectual accuracy for DTM construction.

The results of this study based on different data density are compared in terms of the mean error (ME), the mean 
absolute error (MAE), and the root mean square error (RMSE) with specific reference to the study area

\section{THEORETICAL BACKGROUND}

\subsection{Airborne LiDAR}

The airborne LiDAR is an active remote sensing technique providing its own illumination and measures the ranges (variable distances) to the terrain surface of distant objects. The LiDAR sensor sends out light in the form of a pulsed laser and records the energy scattered back from the terrain surface and the objects on the terrain surface. The range is determined by measuring the round trip time between the light emission and the detection of the reflection (Wehr and Lohr, 1999). Each laser pulse may have multiple returns from features hit at different different ranges from the sensor, creating a cloud of geo-referenced points, including buildings and tree canopy, as well as elevations of bare-earth surface points. Airborne LiDAR is a multi sensor system, consisting of a laser scanner, a Global Navigation Satellite Systems (GNSS) receiver, and an inertial measurement unit (IMU). GNSS is needed to determine the $3 \mathrm{D}$ coordinates of the moving sensor according to one or more differential GNSS base stations. This establishes the origin of each of the thousands of laser pulses emitted each second. IMU directly measures the roll, pitch, and yaw angle of the aircraft in order to determine the angular orientation of the sensor in three dimensions in the flight. The LiDAR sensor measures the scan angle of the laser pulses. Combined with IMU data, this establishes the angular orientation of each of the thousands of pulses emitted each second. The LiDAR sensor also measures the time necessary for each emitted pulse to reflect off the ground (or aboveground features) and return to the sensor. Time translates into distance measured between the aircraft and the point being surveyed (Maune, 2008).

\subsection{Data Reduction Algorithms}

LiDAR based cloud consist of hundreds of millions or billions of sample points, sometimes, requires reduction without losing spatial accuracy while constructing DTMs. Through data reduction, manageable dataset, improved efficiency in storage requirements and processing time can be ensured to achieve an operational and efficient DTM. Many algorithms have been proposed to reduce the $3 \mathrm{D}$ point cloud data in recent years. A good survey on approaches for data reduction is given in Heckbert and Garland (1997). It is beyond the scope of this study to discuss even the most common data reduction algorithms in full detail, though the methods and modifications used within this study is provided.

Uniform data reduction (Lee et al., 2001) uniformly reduces the number of points in the point cloud by subdividing the model space into equally sized cubic cells and deletes all but remained one point from each cell. Random data reduction (Geomagic Support Center, 2014) randomly removes the points based on the specified percentage of the total points that need to be reduced. Each member of the point cloud has an equal chance of being selected (without subjectivity).

\subsection{Interpolation Methods}

The essential data of DTMs are the finite number of points, which have $\mathrm{x} / \mathrm{y}$ coordinates with uniformly spaced z-values. Frequently, the spatial distribution of these points depends on the source of the data. The digital representation of the terrain surfaces via regular or irregular spaced points is possible by an interpolation method. Different interpolation methods applied over the same data may result in different surfaces and hence it is required to evaluate the comparative appropriateness of these techniques. The question of an optimal DTM interpolation method has stimulated several comparative studies, but there is still a lack of consensus about which interpolation method is most appropriate for the terrain data. In this paper, a commonly used interpolation method, Kriging (KRG) method is chosen.

Kriging (KRG) method (Krige, 1951) is a geostatistical and a flexible interpolation method which has been extensively used in diverse fields of mathematics, earth sciences, geography, and engineering and has proved to be powerful and accurate in its fields of use. KRG is also referred to as a linear least squares interpolation (Kraus and Mikhail, 1972) (more specialized to terrain modelling) and it is identical to simple Kriging. According to KRG, both the distance and the degree of variation between reference points are taken into account for optimal spatial prediction (Joseph, 2006). KRG assigns a mathematical function to a certain number of points or all the points located within a certain area of effect in order to determine the output values for each location (Cressie, 1991). KRG uses the semivariogram which describes the variability of data points depending on their distance and direction, and nearby values define the weights that determine the contribution of each data point to the prediction of new values at unsampled locations (Krivoruchko and Gotway, 2004). In case the semi variogram is known, KRG constitutes the best linear unbiased estimator (BLUE) (Yilmaz and Gullu, 2014).

\section{STUDY AREA, SOURCE DATA, AND EVALUATION METHODOLOGY}

Mount St. Helens $\left(46^{0} .1912 \mathrm{~N}, 122^{0} .1944 \mathrm{~W}\right)$ is selected as the study area for the DTM constructions. Mount St. Helens is an active volcano located in Skamania County, Washington, in the Pacific Northwest region of the United States. It is $154 \mathrm{~km}$ south of Seattle, Washington, and $80 \mathrm{~km}$ northeast of Portland, Oregon (Fig. 1). The study area defines approximately area of $116.6 \mathrm{~km}^{2}$. Its span is $\sim 11.9 \mathrm{~km}$ in the north-south direction and $\sim 9.8$ $\mathrm{km}$ in the east-west direction. 


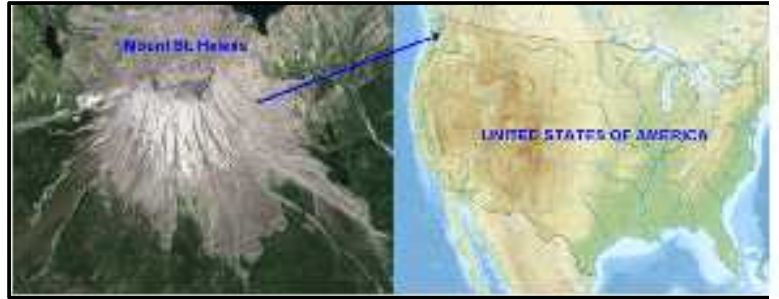

Figure 1. The location of Mount St. Helens

The evaluating procedure of the DTMs refers to an original LiDAR dataset that consisting of 23071760 points $\left(\sim 5.1 \mathrm{~m}^{2} /\right.$ point $)$. The elevation ranges between $743.91 \mathrm{~m}$ and $2539.38 \mathrm{~m}$, with a mean value of 1469.23 $\mathrm{m}$. The data set is contained within the USGS quad Mount St. Helens, WA. The areas of interest flown at different altitudes and scanner settings depending on the area of collection. LiDAR data acquisition specifications are listed below in Table 1. Raw elevation measurements have been determined to be vertically accurate to within $15 \mathrm{~cm}$. LiDAR elevation points are estimated to be horizontally accurate to $0.30 \mathrm{~cm}$. The data set was evaluated against GNSS collected control points which resulted in a vertical RMSE of $0.053 \mathrm{~m}$. LiDAR data were filtered by algorithms within the EarthData's proprietary software and commercial software written by TerraSolid (Mount St. Helens LiDAR Data, 2006).

\begin{tabular}{lll}
\hline & Lower Elevation & Higher Elevation \\
\hline Flying altitude (m) & 2133 & 2438 \\
Flight speed (knots) & 140 & 140 \\
Laser pulse rate (kHz) & 29 & 29 \\
Scan angle (degrees) & \pm 35 & \pm 35 \\
Scan rate (Hz) & 29 & 18 \\
Swath width (m) & 1345 & 1537 \\
\hline
\end{tabular}

Table 1. LiDAR data acquisition specifications

In order to evaluate the effect of the data reduction algorithms on DTM accuracy and to explore the data reduction extent for adequate DTM accuracy; initially, the data density is sequentially reduced through a selection of a predetermined percentage of the original LIDAR data set. Data reduction is performed using the Geomagic Studio ${ }^{\circledR} 12$ software. The original LiDAR data set $(100 \%)$ is reduced to a series of subsets by using uniform, curvature, grid, and random algorithms, representing the $75 \%, 50 \%$, and $25 \%$ of the original LiDAR data set. This reduction protocol is similar to the previous studies of Anderson et al. (2005, 2006). Subsequent to the data reduction, the original LiDAR data set and the reduced data sets are used to produce a series of DTMs. At each data density level, DTMS are constructed via KRG interpolation method.

The evaluation of DTM accuracy is focused on the correspondent elevation differences between the reference DTM (based on the original dataset) and the test DTMs (based on the reduced datasets) using the equation below:

$\Delta Z=Z_{(100 \%)}-Z_{(i \%)}$

(1) where $\Delta Z$ is the elevation residual, $Z$ is the elevation value of nodes estimated from (reference and test) DTMs, and $i$ represents the data density ( $\mathrm{i}=75,50$ and 25).

For the statistical analysis of elevation differences, minimum and maximum values of $\Delta Z$ are determined and the overall performance of DTMs is assessed through ME, MAE, and RMSE accuracy measures defined by:

$$
M E=\frac{1}{n} \sum_{k=1}^{n} \Delta Z
$$

$$
M A E=\frac{1}{n} \sum_{k=1}^{n}|\Delta Z|
$$

$$
R M S E=\sqrt{\frac{1}{n} \sum_{k=1}^{n}(\Delta Z)^{2}}
$$

(4) where $n$ is the number of the points used for the accuracy verification and $k$ refers to the residual sequence. $\mathrm{ME}$ is a measure of underestimation or overestimation the true value of the interpolation method. MAE provides the average deviation that DTM surface deviates from the true value to measure the effect of the data reduction on DTM accuracy. RMSE is calculated to measure the overall accuracy of DTM surface.

\section{CASE STUDY}

For the evaluation process, the reference DTM of the study (Fig. 2) is constructed from the original LiDAR dataset using KRG method, implemented within the Surfer ${ }^{\circledR} 13$ software. The accuracy of the reference DTM is assessed through cross-validation technique.
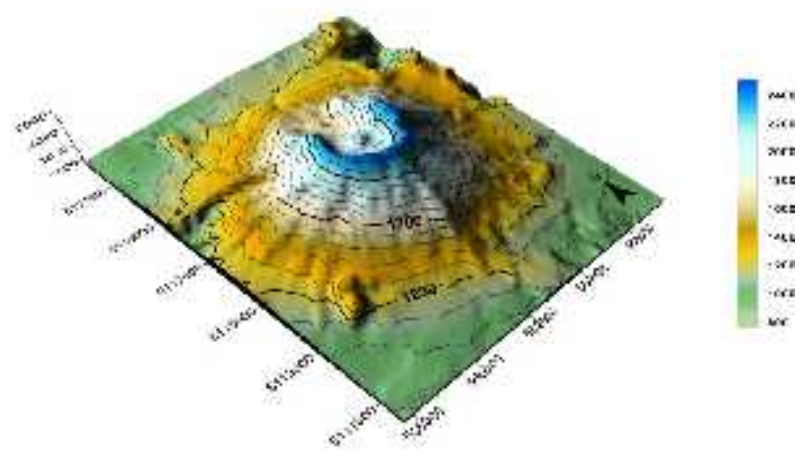

Figure 2. Reference DTM of the study area

The reduced data sets, based on uniform and random algorithms, are used to construct the test DTMs of the study area using KRG method, at each data density level $(75 \%, 50 \%$, and $25 \%)$. The test DTMs (DTMi $\% ; i=75$, 50 , and 25) are subtracted from the corresponding reference DTMs (DTM100\%) for quantifying elevation differences. Also, the graphical representations have been adopted for the comparative evaluation of the test DTMs by producing a residual map for each test DTM 
(Fig. 3) that indicates the occurrence and magnitude of elevation differences (Weng, 2006), in relation to terrain characteristics (by overlaying the contour map of Mount St. Helens on the residual maps).
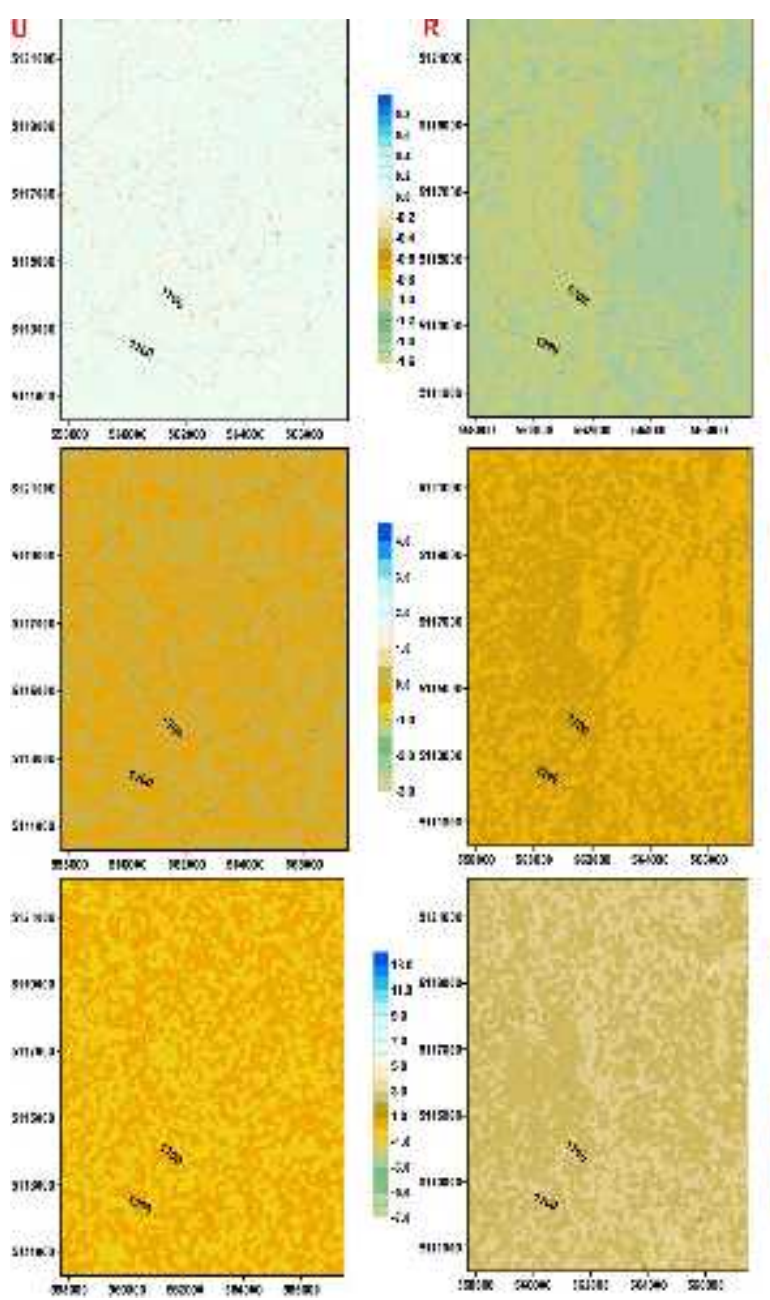

Figure 3. Residual maps of test DTMs for the study area (upper row: 100-75; middle row: 100-50; lower row: 100-75)

\section{COMPARATIVE RESULTS AND CONCLUSIONS}

The visual analysis of the elevation residual maps shows that the deviation of the test DTMs from the reference DTM are getting smaller depending on the increase in data density, for both data reduction algorithms. From the visual interpretation of the elevation residual maps, it is evident that uniform algorithm gave better results than random algorithm.

Global statistics of elevation residuals based on uniform and random algorithms with KRG method at selected data density levels are presented in Table 2 . When the statistics summarized in Table 2 are evaluated, it can be concluded that uniform algorithm provides more accurate results than random algorithm at all data densities.
Table 2. Statistics of the elevation residuals over the study area

\begin{tabular}{lcccccc}
\hline & \multicolumn{3}{c}{ UNIFORM } & \multicolumn{3}{c}{ RANDOM } \\
\cline { 2 - 6 } & $75 \%$ & $50 \%$ & $25 \%$ & $75 \%$ & $50 \%$ & $25 \%$ \\
\hline Min & - & - & -6.964 & -3.106 & -7.943 & - \\
& 1.56 & 2.83 & & & & 11.58 \\
& 9 & 7 & & & & 7 \\
Max & 0.88 & 3.56 & 12.07 & 11.59 & 12.38 & 12.66 \\
& 3 & 7 & 1 & 2 & 3 & 5 \\
ME & - & 0.00 & 0.006 & -0.005 & -0.003 & -0.018 \\
& 0.00 & 2 & & & & \\
& 1 & & & & & \\
MAE & 0.02 & 0.05 & 0.126 & 0.112 & 0.154 & 0.239 \\
& 1 & 8 & & & & \\
RMS & 0.06 & 0.14 & 0.340 & 0.266 & 0.352 & 0.531 \\
E & 2 & 9 & & & & \\
\hline
\end{tabular}

MEs are recorded at the centimetre level at $25 \%$ data density for uniform and random data reduction algorithms $(0.006 \mathrm{~m}$. and $-0.018 \mathrm{~m}$.). KRG underestimated the terrain surface because of the predominantly concentric topography of the study area. MEs are sub-centimeter at $50 \%$ and $75 \%$ data densities for uniform and random data reduction algorithms, indicating that interpolation biases were negligible.

Throughout the decreasing data densities, the test DTMs have increasing MAEs ranging from $0.021 \mathrm{~m}$ to 0.239 $\mathrm{m}$. Terrain representations derived from the test DTMs based on uniform algorithm are better than the test DTMs based on random algorithms, at all data densities.

RMSEs ranged from $0.062 \mathrm{~m}$. to $0.531 \mathrm{~m}$. show significant increases for uniform and random data reduction algorithms as data densities decreased from $75 \%$ to $25 \%$. As expected, the lowest RMSEs are obtained at $75 \%$ data density level. RMSEs of the test DTMs for data reduction algorithms have a decreasing sequence as uniform $<$ curvature, at all data densities.

In terms of overall accuracy, there is no significant decrease for the test DTMs constructed from high data densities $(75 \%$ and 50\%). Hence, it becomes apparent that the test DTMs based on $75 \%$ and $50 \%$ point densities are sufficient for terrain representations

Based on the analysis results of comparison of data reduction algorithms in constructing DTMs, the following conclusions can be drawn based on this paper: (i) Uniform data reduction algorithm can be considered as a feasible technique due to better terrain representation for constructing LiDAR-derived DTMs. (ii) KRG biases are negligible with lower RMSEs in terms of grid data reduction approach at higher data densities $(75 \%$ and $50 \%$ ). (iii) LiDAR datasets can be reduced to $50 \%$ density level while still maintaining the DTM accuracy.

Due to advancements and improvements in instrumentation, software, processes, applications, and understanding, airborne LiDAR is one of the most capable, effective, and reliable alternative to traditional systems for gathering high-accuracy and high-density 3D terrain data leading to mapping products. The 
limitations of the use of LiDAR data in constructing DTMs are the magnitude of data and the intense postprocessing that can be required to transform raw LiDAR data into point clouds and eventually DTMs and other data products. However, high-density data associated with LiDAR lead to imposing challenges with respect to data storage, processing and manipulation. Large data volumes procured from LiDAR often require data reduction without losing relevant geometric details while constructing DTMs because the quality of LiDARderived DTMs equates to how well it represents the terrain undulation and continuity. Ultimately, the results of this study show that uniform algorithm is favoured for data reduction due to its lowest RMSEs and MAEs. The identification of terrain feature points is important because all data points do not contribute optimally to the DTM accuracy. Therefore, the data should be reduced by keeping critical data (considering terrain features). Due to the required DTM accuracy, extensive attention should be paid to reducing LiDAR data without extraction critical terrain elements. In order to represent the terrain morphology with the reduced data, further studies using diverse data reduction algorithms for smaller data density intervals (in relation to landform types) are necessary to determine the effective data reduction algorithm and the threshold data density for constructing DTMs.

\section{ACKNOWLEDGEMENTS}

This study was supported by Afyon Kocatepe University Scientific Research Projects Coordination Department (Project No: 16.KARIYER.87).

\section{REFERENCES}

Aguilar, F.J., Agüera, F., Aguilar, M.A., Carvajal, F. 2005. Effects of terrain morphology, sampling density and interpolation methods on grid DEM accuracy. Photogrammetric Engineering and Remote Sensing, 71 (7), 805-816.

Aguilar, F.J., Aguilar, M.A., Agüera, F., 2007. Accuracy assessment of digital elevation models using a nonparametric approach. International Journal of Geographical Information Science, 21 (6), 66-686.

Aguilar, F.J., Mills, J.P. 2008. Accuracy assessment of LiDAR-derived digital elevation models. The Photogrammetric Record, 23 (122), 148-169.

Anderson, E.S., Thompson, J.A., Austin, R.E. 2005 LIDAR density and linear interpolator effects on elevation estimates. International Journal of Remote Sensing, 26 (18), 3889-3900.

Anderson, E.S., Thompson, J.A., Crouse, D.A., Austin, R.E. 2006. Horizontal resolution and data density effects on remotely sensed LIDAR-based DEM. Geoderma, 132 406- 415 .

Arab-Sedze, M., Heggy, E., Bretar, F., Berveiller, D., Jacquemoud, S. 2014. Quantification of L-band InSAR coherence over volcanic areas using LiDAR and in situ measurements. Remote Sensing of Environment, 152, 202-216.
Arun, P.V. 2013. A comparative analysis of different DEM interpolation methods. The Egyptian Journal of Remote Sensing and Space Sciences, 16, 133-139.

Cavalli, M., Tarolli, P. 2011. Application of LiDAR technology for rivers analysis. Italian Journal of Engineering Geology and Environment, Special Issue (1), 33-44.

Chaplot, V., Darboux, F., Bourennane, H., Leguédois, S., Silvera, N., Phachomphon, K. 2006. Accuracy of interpolation techniques for the derivation of digital elevation models in relation to landform types and data density. Geomorphology, 77, 126-141.

Chen, C.F., Yue, T.X. 2010. A method of DEM construction and related error analysis. Computers and Geosciences, 36 (6), 717-725.

Chu, H.J., Chen, R.A., Tseng, Y.H., Wang, C.K. 2014. Identifying LiDAR sample uncertainty on terrain features from DEM simulation, Geomorphology, 204, 325-333.

Cressie, N.A.C. 1991. Statistics for Spatial Data. New York: John Wiley and Sons.

Dorn, H., Vetter, M., Höfle, B. 2014. GIS-based roughness derivation for flood simulations: a comparison of orthophotos, LiDAR and crowdsourced geodata. Remote Sensing, 6, 1739-1759.

Erdogan, S. 2010. Modelling the spatial distribution of DEM error with geographically weighted regression: an experimental study. Computers and Geosciences, 36, 34-43.

Fassnacht, F.E., Hartig, F., Latifi, H., Berger, C., Hernández, J., Corvalán, P., Koch, B. 2014. Importance of sample size, data type and prediction method for remote sensing-based estimations of aboveground forest biomass. Remote Sensing of Environment, 154, 102-114.

Fisher, P.F., Tate, N.J. 2006. Causes and consequences of error in digital elevation models. Progress in Physical Geography, 30 (4), 467-489.

Garnero, G., Godone, D. 2013. Comparisons between different interpolation techniques. International Archives of the Photogrammetry, Remote Sensing and Spatial Information Sciences. XL-5/W3, 139-144.

Geomagic Support Center 2014. Overview of the Point Sampling Commands. <support1.geomagic.com>

Gong, J., Li, Z., Zhu, Q., Sui, H., Zhou, Y. 2000. Effects of various factors on the accuracy of DEMs: an intensive experimental investigation. Photogrammetric Engineering and Remote Sensing, 66 (9), 1113-1117.

Gumus, K., Sen, A. 2013. Comparison of spatial interpolation methods and multi-layer neural networks for different point distributions on a digital elevation model. Geodetski Vesnik, 57 (3), 523-543. 
Heckbert, P., Garland, M. 1997. Survey of polygonal surface simplification algorithms. Proceedings of the 24th Annual Conference on Computer Graphics and Interactive Techniques, SIGGRAPH '97, pp. 209-216.

Hodgson, M.E., Bresnahan, P. 2004. Accuracy of airborne LiDAR-derived elevation: empirical assessment and error budget. Photogrammetric Engineering \& Remote Sensing, 70 (3), 331-339.

Immelman J., Scheepers L.G.C. 2011. The effects of data reduction on LiDAR-based digital elevation models, 4th International Congress on Image and Signal Processing, Shanghai, China, 1694-1698.

Joseph, V.R. 2006. Limit Kriging. Technometrics, 48 (4), 458-466.

Kraus, K., Mikhail, E. 1972. Linear least squares interpolation. Photogrammetric Engineering, 38, 10161029.

Krige, D.G. 1951. A statistical approach to some basic mine valuation problems on the Witwatersrand. Journal of the Chemical, Metallurgical and Mining Society of South Africa, 52 (6), 119-139.

Krivoruchko, K., Gotway, C.A. 2004. Creating exposure maps using Kriging. Public Health GIS News and Information, 56, 11-16.

Lee, K.H., Woo, H., Suk, T. 2001. Point data reduction using 3D grids. The International Journal of Advanced Manufacturing Technology, 18 (3), 201-210.

Li, Z., Zhu, C., Gold, C. 2005. Digital Terrain Modeling: Principles and Methodology. Boca Raton: CRC Press.

Liu, X., Zhang, Z., Peterson, J., Chandra, S. 2007. The effect of LiDAR data density on DEM accuracy. International Congress on Modelling and Simulation: Land, Water and Environmental Management: Integrated Systems for Sustainability, Christchurch, New Zealand, 10-13 December 2007, pp. 1363-1369.

Liu, X. 2008. Airborne LiDAR for DEM generation: some critical issues. Progress in Physical Geography, 31 (1), 31-49.

Liu, X., Zhang, Z. 2008. LiDAR data reduction for efficient and high quality DEM generation, The International Archives of the Photogrammtery, Remote Sensing and Spatial Information Sciences, XXXVII, 173-178.

Liu, H., Kiesel, J., Hörmann, G., Fohrer, N. 2011. Effects of DEM horizontal resolution and methods on calculating the slope length factor in gently rolling landscapes. Catena, 87 (3), 368-375.

Ma, R., Meyer, W. 2005. DTM generation and building detection from LiDAR data. Photogrammetric Engineering and Remote Sensing, 71, 847-854.

Maune, D.F., Kopp, S.M., Crawford, .A., Zervas, C.E. 2007. Introduction. In D.F. Maune (Ed.), Digital
Elevation Model Technologies and Applications: The DEM Users Manual (2nd ed.) (pp. 1-36). Bethesda: American Society for Photogrammetry and Remote Sensing.

Maune, D.F. 2008. Aerial mapping and surveying. In S.O. Dewberry, and L.N. Rauenzahn (Eds.), Land Development Handbook (3rd ed.) (pp. 877-910). New York: McGraw-Hill.

Mount St. Helens LiDAR Data 2006. $<$ https://wagda.lib.washington.edu/data/type/elevation/li dar/st_helens/>

Mukherjee, S., Joshi, P.K., Mukherjee, S., Ghosh, A., Garg, R.D., Mukhopadhyay, A. 2013. Evaluation of vertical accuracy of open source digital elevation model (DEM). International Journal of Applied Earth Observation and Geoinformation, 21, 205-217.

Polat, N., Uysal, M. 2015. Investigating performance of Airborne LiDAR data filtering algorithms for DTM generation. Measurement, 63, 61-68.

Rayburg, S., Thoms, M., Neave, M. 2009. A comparison of digital elevation models generated from different data sources. Geomorphology, 106, 261-270.

Razak, K.A., Straatsma, M.W., van Westen, C.J., Malet, J.P., de Jong, S.M. 2011. Airborne laser scanning of forested landslides characterization: Terrain model quality and visualization. Geomorphology, 126, 186200.

Renslow, M.S. 2012. Introduction. In M.S. Renslow (Ed.), Manual of Airborne Topographic LiDAR (pp. 15). Bethesda: ASPRS.

Sailer, R., Rutzinger, M., Rieg, L. Wichmann, V. 2014. Digital elevation models derived from airborne laser scanning point clouds: appropriate spatial resolutions for multi-temporal characterization and quantification of geomorphological processes. Earth Surface Processes and Landforms, 39 (2), 272-284.

Tan, Q., Xu, X. 2014. Comparative analysis of spatial interpolation methods: an experimental study. Sensors and Transducers, 165 (2), 155-163.

Tarolli, P., Arrowsmith, J.R., Vivoni, E.R. 2009. Understanding earth surface processes from remotely sensed digital terrain models. Geomorphology, 113, 1-3.

Vianello, A., Cavalli, M., Tarolli, P. 2009. LiDARderived slopes for headwater channel network analysis. Catena, 76 (2), 97-106.

Wehr, A., Lohr, U. 1999. Airborne laser scanning-An introduction and overview. ISPRS Journal of Photogrammetry and Remote Sensing, 54, 68-82.

Weng, Q. 2006. An evaluation of spatial interpolation accuracy of elevation data. In A. Riedl, W. Kainz, and G.A. Elmes (Eds.), Progress in Spatial Data Handling (pp. 805-824). Berlin: Springer-Verlag. 
Yan, W.Y., Shaker, A., El-Ashmawy, N. 2015. Urban land cover classification using airborne LiDAR data: A review. Remote Sensing of Environment, 158, 295-310.

Yilmaz, M., Gullu, M. 2014. A comparative study for the estimation of geodetic point velocity by artificial neural networks. Journal of Earth System Sciences, 123 (4), 791-808.
Copyright (C) International Journal of Engineering and Geosciences (IJEG). All rights reserved, including the making of copies unless permission is obtained from the copyright proprietors. 